\title{
Laboratory analogues of hydrocarbonated interstellar nanograins
}

\author{
T. Pino ${ }^{1}$, A. T. Cao ${ }^{1}$, Y. Carpentier ${ }^{1}$, E. Dartois ${ }^{2}$, L. d'Hendecourt ${ }^{2}$, \\ and Ph. Bréchignac ${ }^{1}$ \\ ${ }^{1}$ Laboratoire de Photophysique Moléculaire, UPR 3361, Université Paris-Sud, \\ 91405 Orsay Cedex, France \\ email: philippe.brechignac@u-psud.fr \\ ${ }^{2}$ Institut d'Astrophysique Spatiale, UMR-8617, Université Paris-Sud, \\ 91405 Orsay Cedex, France
}

\begin{abstract}
Carbonaceous extraterrestrial matter is observed in a wide variety of astrophysical environments. The spectroscopic signatures revealed a large variety of chemical structure illustrating the rich carbon chemistry that occurs in space. In order to produce laboratory analogues of carbonaceous cosmic dust, a new chemical reactor has been built in the Laboratoire de Photophysique Moléculaire. It is a low pressure flat burner providing flames of premixed hydrocarbon / oxygen gas mixtures, closely following the model system used by the combustion community. In such a device the flame is a one-dimensional chemical reactor that offers a broad range of combustion conditions and sampling which allows production of many and various byproducts. In the present work, we have studied: i) the infrared transmission spectra of thin film deposit samples whose nature ranges from strongly aromatic to strongly aliphatic materials; ii) the resonant two-photon photoionisation spectra of gas phase PAHs formed in the flame.
\end{abstract}

Keywords. Astrochemistry, methods: laboratory, ISM: dust, molecules

\section{Introduction}

The ubiquitous presence of complex organic matter in many and various astrophysical environments is a relatively recent observational fact. More specifically, carbon chains, large aromatic-like clusters or molecules (PAHs), hydrogenated amorphous carbon, and diamond are materials that are directly observed or thought to be components of the interstellar medium (ISM). Most importantly it is now widely accepted that interstellar "nanograins" having sizes intermediate between those of the PAHs easily accessible to laboratory studies and those of the "standard" interstellar grains (ca $50 \mathrm{~nm}$ ) are ubiquitous "free-flyers" in ISM.

These nanograins await characterization of their intimate structures, therefore laboratory analogues of these particles have to be spectroscopically characterized in the gas phase. This is the goal of the new experimental set-up, recently built at the Laboratoire de Photophysique Moléculaire, devoted to the production and characterization of such carbonaceous nanoparticles using a hydrocarbon-rich, premixed, flat, low-pressure, hydrocarbon / oxygen flame. The mass distribution of products in the reactor is monitored by a high resolution Time-Of-Flight spectrometer, after sampling the flame through a quartz cone. The spectroscopic properties can be measured both in absorption in solid phase, using thin films deposits, and in the gas phase using laser techniques. The results obtained so far from such measurements have revealed a strong evolution from aromatic materials containing aliphatic substituents to large polymer-like soot particles. 


\section{Results}

Excellent control of the combustion conditions and of the sampling has been demonstrated. Consequently, by tuning the operating conditions of our particle source we can produce specific astrophysically relevant analogues. Spectroscopic studies on the infrared transmission of thin films have allowed us to propose a coherent scenario (Pino et al. $2008)$ to explain the shift in the position of the "6.2-6.3 $\mu \mathrm{m}$ band" among the aromatic emission features observed in various astrophysical environments ranging from "Class A" to "Class C" sources (Peeters et al. 2002).

In the gas phase we can obtain spectroscopic characterization of the species present in the sampled part of the flame thanks to two-photon resonant photoionisation laser spectroscopy. An example of such spectra is shown in Figure 1, where three different aromatic species were simultaneously monitored. In each case the pattern of the resonant $S_{1} \leftarrow S_{0}$ electronic transition gives access to the unambiguous identification of a given molecule. This technique is extremely useful for discriminating between specific chemical structures in the case of isomers. Such new data open the way to entirely novel spectra of large PAHs in the gas phase.



Figure 1. Resonant 2-photon photoionisation spectroscopy of a few aromatic molecules produced in the flame.

In conclusion we have shown that our new apparatus has proven its ability to form astrophysically relevant laboratory analogues of organic interstellar dust, from which further work should help to elucidate in the near future some of the present key questions about the evolution of organic matter in space, from the diffuse interstellar clouds to disks and planets.

\section{References}

Peeters, E., Hony, S., van Kerckhoven, C., et al. 2002 A\&A, 390, 1089

Pino, T., Dartois, E., Cao, A. T., Carpentier, Y., Chamaillé, T., Vasquez, R., Jones, A., d'Hendecourt, L., \& Bréchignac, Ph. 2008, A\& A, submitted 